\title{
ESTIMACIÓN DEL VOLUMEN ORBITARIO MEDIANTE IMÁGENES DE TC Y EL PRINCIPIO DE CAVALIERI
}

\author{
Drs. César Coronado $G^{(1)}$, Oscar Arriagada $C^{(1)}$, Daniela Zavando $M^{(2)}$, Mariano del Sol $C^{(3)}$, Iván Suazo $G^{(1)}$.
}

1. Departamento de Ciencias Básicas Biomédicas, Universidad de Talca, Chile.

2. Universidad Autónoma de Chile, sede Talca, Chile.

3. Facultad de Medicina, Universidad de La Frontera, Chile.

\section{ESTIMATION OF THE ORBITAL VOLUME WITH CT IMAGES AND CAVALIERI'S PRINCIPLE}

\begin{abstract}
Determination of the orbital volume has significance in cases such as facial injuries and lens implants where it is necessary to restore the normal position of the structures, since providing the patient with a volume-deficient orbit may result in enophthalmos. The aim of this study is to assess the volume of the orbit by using the Cavalieri's principle supplemented with sequential analysis of CT images on the computer screen. We conducted a series of CT images with a thickness of $1 \mathrm{~mm}$ on 10 human skulls. After selection, initially random and then sequential, images were analyzed on a computer screen with a mesh test chart to determine orbital area. Once having identified both area and thickness, we measured the volume of segments, and the total volume of the orbit was extrapolated by using a mathematical formula based on the Cavalieri's principle.

Results were compared with the actual volume obtained by the displacement of fluid from printouts of the orbits. The result of this comparison suggests that the method is statistically reliable when considering that we assessed seven images of a randomly chosen orbit and then in a known sequence with a straightforward technique for orbital volume estimation.

Keywords: Cavalieri's Principle, Computed Tomography, Images, Orbit, Volume.
\end{abstract}

Coronado $\boldsymbol{C}$ y cols. Estimación del volumen orbitario mediante imágenes de TC y el principio de Cavalieri. Rev Chil de Radiol 2010; 16(2): 59-63.

Correspondencia: Prof. Dr. Iván Suazo G.

Depto. de Ciencia Básicas Biomédicas. Fac. de Ciencias de la Salud, Universidad de Talca

Av. Lircay s/n oficina 104, Talca-Chile.

isuazo@utalca.cl

Trabajo recibido el 03 de abril de 2010, aceptado para

publicación el 18 de mayo de 2010.
Resumen: Conocer el volumen de la órbita es importante en traumatismos faciales o implantes oculares, donde es necesario restablecer la posición normal de las estructuras y devolver un volumen deficiente puede traducirse en enoftalmo. El objetivo del presente trabajo es realizar estimación del volumen orbitario utilizando el principio de Cavalieri complementado con análisis secuencial de imágenes de TC en la pantalla del computador. Se realizó imágenes de TC de $1 \mathrm{~mm}$ de espesor a 10 cráneos humanos. Luego de la selección, inicialmente aleatoria y luego secuencial, las imágenes se analizaron sobre la pantalla del computador con un test de malla de puntos para determinar área orbitaria. Conociendo el área y el grosor, determinamos el volumen de los segmentos y se extrapola al volumen total de la órbita, utilizando fórmula matemática basada en el principio de Cavalieri.

Los resultados fueron comparados con el volumen real obtenido por el desplazamiento de fluidos de impresiones de las órbitas. Esta comparación sugiere que el método es estadísticamente confiable al evaluar 7 imágenes de una órbita escogida aleatoriamente y luego en una secuencia conocida, con una manera simple de estimación del volumen.

Palabras clave: Orbita, Principio de Cavalieri, Tomografía computada, Volumen.

\section{Introducción}

Las órbitas son cavidades de forma piramidal, ubicadas en el centro de la región craneofacial ${ }^{(1)}$. Su ubicación, una transición entre neurocráneo y esplacnocráneo, hace que generalmente se vean comprometidas en los traumatismos faciales $\left(40 \%{ }^{(2)}\right.$; estos traumatismos modifican la estructura de la órbita, alterando su capacidad volumétrica ${ }^{(3)}$. También existe un gran número de alteraciones de tipo congénito, neoplásico, vascular y endocrino que pueden alterar significativamente el crecimiento de la órbita ${ }^{(4)}$.

La evaluación del volumen de la órbita es importante en la planificación de la rehabilitación 
en traumatismos, donde la posición de las paredes orbitarias se encuentra alterada, lo que sumado a la modificación de la posición de los tejidos blandos hacen del enoftalmo una complicación frecuente ${ }^{(7)}$. Esta estimación también es necesaria para la planificación que se efectúa previo a la colocación de implantes oculares ${ }^{(8)}$.

Es posible determinar el volumen de la órbita utilizando software asociados a los programas de visualización de las imágenes. Sin embargo, su manejo requiere la participación de un operador especializado. Otro método para determinar el volumen de la órbita consiste en utilizar imágenes seriadas de tomografía computada (TC) ${ }^{(3,5,7)}$.

Un método sencillo que permite estimar volúmenes a partir de imágenes es el basado en el principio de Cavalieri, que consiste básicamente en la determinación del área aproximada en una imagen de TC, para luego relacionarla con el grosor del corte y así obtener un volumen de dicho corte. El principio de Cavalieri permite calcular el volumen de cualquier estructura de forma irregular, seccionada de manera sistemática, si se conocen los valores del área que ocupa cada corte y el grosor de ellos. La fórmula se expresa en la Figura 1. En el caso de imágenes de TC de órbita, se calculará el área de cada corte, lo que nos permitirá calcular el volumen total. Este procedimiento puede ser realizado sobre imágenes impresas de $\mathrm{TC}^{(8)}$, en donde la relación entre tamaño aparente y tamaño real se incorpora a los cálculos.

\section{$V$ cuerpo $=\Sigma V$ de los segmentos del cuerpo}

Figura 1. Fórmula que representa el principio de Cavalieri, que se basa en conocer el área de un segmento (base) y grosor del segmento (altura) para multiplicarlos y obtener el volumen del segmento. El volumen del cuerpo es la sumatoria de los volúmenes de los segmentos.

Cuando se trata de estructuras voluminosas, como la órbita, seno maxilar, etc., o cuando el grosor del corte programado es pequeño (1-2 $\mathrm{mm}$ ), la aplicación del principio de Cavalieri a las imágenes médicas presenta el inconveniente que resulta complicado determinar el área de todas las imágenes en las que aparece la estructura cuyo volumen queremos estimar. Ante este problema, la estereología nos aporta algunas herramientas útiles, que nos permiten determinar sin sesgo el volumen de una estructura irregular, combinando el principio de Cavalieri con la estadística, de modo que una estructura seccionada mediante un número de cortes $\mathrm{K}$, permita estimar el volumen al conocer el área que ocupan en la imagen un número $\mathrm{K} / \mathrm{y}$ de cortes.

En el presente trabajo presentamos un método de estimación del volumen orbitario utilizando el principio de Cavalieri, simplificado mediante el uso de la estadística y la medición sobre la pantalla del computador.

\section{Material y métodos}

Se seleccionaron 10 cráneos secos de individuos adultos, pertenecientes al Laboratorio de Morfología de la Universidad de Talca, que fueron colocados en cajas de aislapol, para su estabilización en posición anatómica, y llevados al tomógrafo Toshiba Asteion 4 , multicorte de cuatro canales. Se realizó una serie de imágenes de TC de un espesor de $1 \mathrm{~mm}$, con la finalidad de estimar el volumen de la órbita derecha e izquierda, basándose en el principio de Cavalieri.

Las imágenes fueron obtenidas por el tomógrafo (adquisición volumétrica) y visualizadas en un computador anexo al equipo, donde fueron grabadas en un disco compacto para su posterior traslado y análisis. Las imágenes se observaron con el visualizador de imágenes de Windows para Windows XP versión Service Pack 2 con una resolución de pantalla de 1280 x 800; superpuesta sobre la pantalla del computador se utilizó un sistema de test de malla de puntos cubriendo la totalidad de la imagen. La malla test se construyó sobre una lámina transparente impresa con puntos ubicados de manera ordenada a una distancia constante de $5 \mathrm{~mm}$ entre sí (Figura 2).

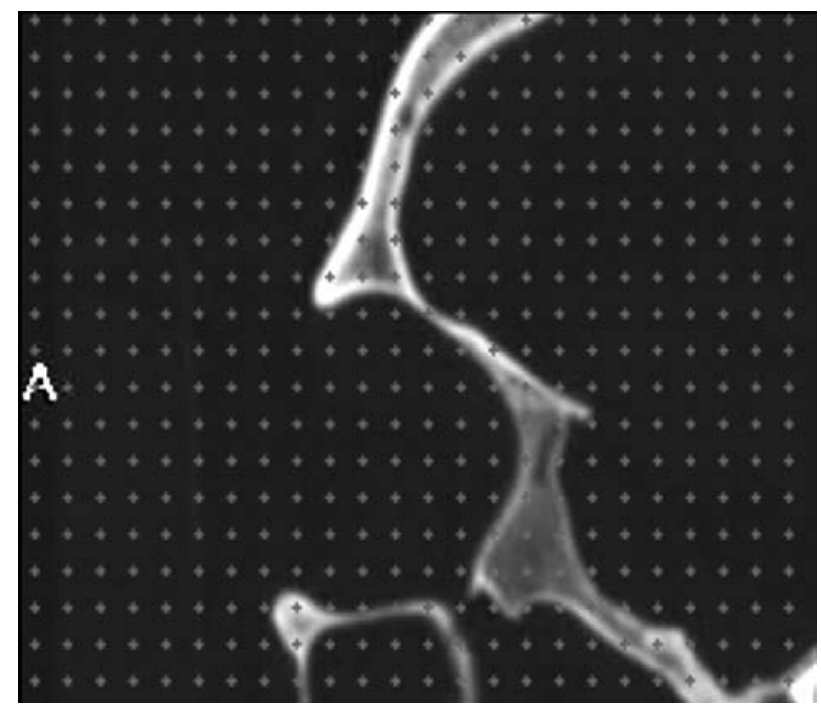

Figura 2. Esquema de una imagen de TC, donde la órbita está cubierta en su totalidad por una malla de puntos separados por $5 \mathrm{~mm}$.

Las imágenes fueron observadas y de ellas se seleccionaron las que contenían algún segmento de las órbitas derecha e izquierda. Para la realización de los cálculos se contaron los puntos que se encontraron dentro del área anatómica correspondiente a 
las órbitas, lo que nos entregó un área estimada del segmento.

Para la elección de la cantidad de imágenes a analizar, se separó en grupos el total de imágenes en que se observaba la órbita. Aleatoriamente, se seleccionó la primera imagen a observar para luego seguir la secuencia, dependiendo del número de cortes que presentaran los grupos.

Para calcular el volumen estimado, se utilizó la fórmula modificada de estimación de volumen de imágenes radiológicas ${ }^{(9)}$ :

$$
V=\frac{g \times \quad[(U E) \times d]^{2}}{(L E)} \times \Sigma P \times C G
$$

$g$ = Grosor de la sección consecutiva.

$\mathrm{UE}=$ Unidad de escala de la imagen observada en computador.

$\mathrm{d}=$ Distancia entre los puntos de la malla de test.

LE $=$ Longitud medida de la escala sobre la pantalla de computador.

$\sum \mathrm{P}=$ Sumatoria de los puntos contabilizados en todas las imágenes observadas.

$\mathrm{CG}=$ Corresponde al número de cortes por grupo.

Ejemplo: Cráneo X: al observar las imágenes en el computador se determinó que la órbita derecha se encontraba desde el corte 7 al 45 de la serie de cortes. Se seleccionaron los 38 cortes en 7 grupos de 5 cortes y 1 grupo de 3 cortes (sobrante): $45-7=38$, $38 / 5=7$ ( 3 es el sobrante). Con los grupos conformados se selecciona aleatoriamente un corte del 1 al 5 , ejemplo: el 4, que será nuestro punto de partida de donde luego se analizarán secuencialmente cada 5 cortes a partir del corte 4 . Del total de imágenes que analizamos, contamos 98 puntos. Determinamos la medida en centímetros que entrega la TC $(20 \mathrm{~mm})$ con una regla milimetrada $(14 \mathrm{~mm})$, quedando establecidas las variables de la fórmula:

$\mathrm{g}=1 \mathrm{~mm}$

$\mathrm{UE}=20 \mathrm{~mm}$

$\mathrm{d}=5 \mathrm{~mm}$

$\mathrm{LE}=14 \mathrm{~mm}$

$\sum \mathrm{P}=98$

$\mathrm{CG}=5$

\section{Determinación del volumen real (Gold Standard)}

El volumen real de las órbitas se obtuvo de una impresión de las cavidades orbitarias, utilizando como material de impresión la silicona pesada de consolidación por condensación (Polidimetil siloxano), de uso odontológico (swiss tec $®$ putty). Para la toma de impresión fue necesario el sellado de las cavidades anatómicas dentro de las órbitas, que fue realizado con cera amarilla de uso odontológico. El registro impreso en silicona fue medido bajo el principio de Arquímedes, también conocido como la técnica de desplazamiento de fluidos. Para este propósito, cada impresión de órbita fue colocada en un matraz de aforo de $90 \mathrm{ml}$, con agua a temperatura ambiente; el desplazamiento de agua muestra el volumen de la impresión de la órbita.

\section{Plan de análisis}

Se analizaron grupos de imágenes de 3, 5 y 9 cortes, de manera bilateral, calculándose el volumen orbitario estimado al aplicar la fórmula descrita. Los valores obtenidos fueron comparados con los del volumen real, estableciéndose el porcentaje de error entre el volumen real y el calculado, si las diferencias eran estadísticamente significativas, utilizando para ello $T$ test para muestras relacionadas con $p<0,05$; la correlación de Pearson entre el volumen real y los volúmenes calculados con 3, 5 y 9 cortes.

\section{Resultados}

El número de cortes que incluía el segmento de órbita fue variable. Se estimó el volumen orbitario a partir de grupos de 3, 5 y 9 cortes; el número de imágenes analizadas varió en relación al tamaño de la órbita y al número de cortes que incluyó algún segmento de órbita, como se observa en la Tabla I.

Nuestros resultados indican que en ninguno de los tres grupos la estimación del volumen fue significativamente distinta del volumen real (gold standard), el porcentaje de error fue bajo (rango $-6,05$ a 3,23\%), con una alta correlación. El detalle de estos resultados se encuentra en la Tabla I y se observa en las Figuras 3 y 4.

$$
V=1 \mathrm{~mm} \times \frac{[(20 \times 5 \mathrm{~mm})]^{2}}{14 \mathrm{~mm}} \times 98 \times 5=24996,997 \mathrm{~mm}^{3}=24,996 \mathrm{~cm}^{3}
$$

El volumen de la órbita derecha del cráneo $X$ es de $25 \mathrm{~cm}^{3}$ aproximadamente. 
Tabla I. Datos estadísticos descriptivos y analíticos de los volúmenes real y estimados a partir de secuencias de grupos de 3,5 y 9 cortes.

Desv. Error* Significativo** Correlación

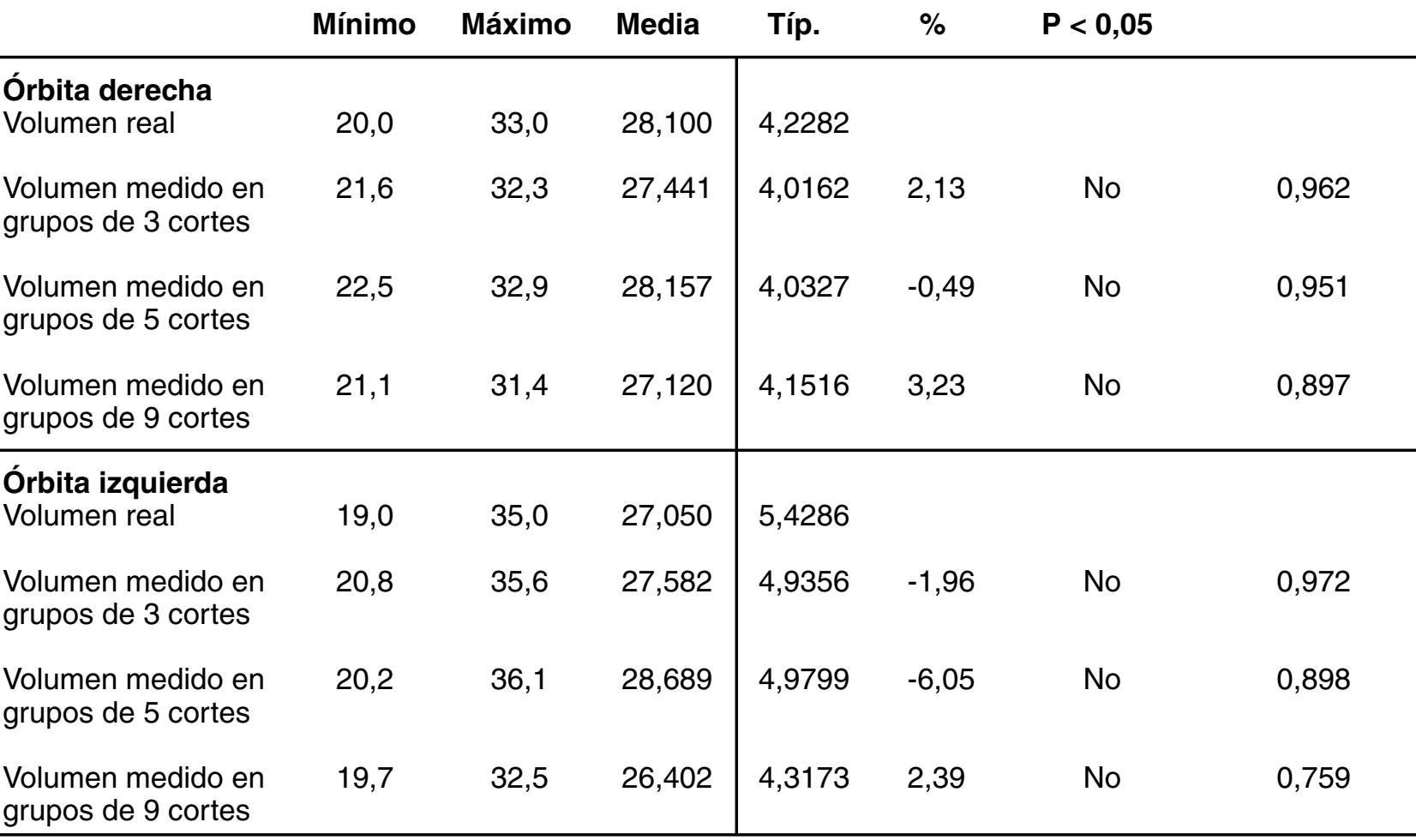

* Porcentaje de error con respecto al volumen real, los valores negativos indican que el valor obtenido es mayor que el gold standard.

** Significancia estadística al comparar con el valor real mediante one way ANOVA con Bonferroni post test.

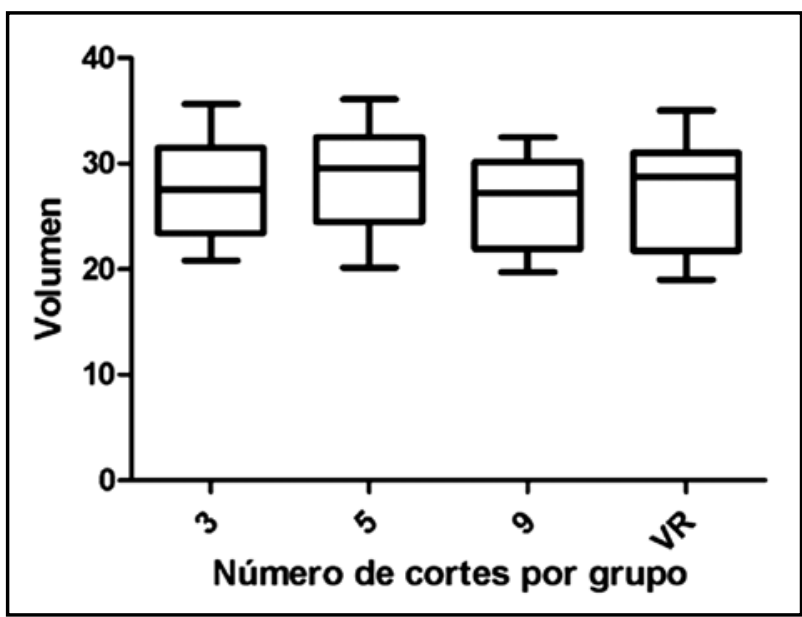

Figura 3. Diagrama de caja, que muestra el número de cortes $\mathrm{v} / \mathrm{s}$ volumen real de la órbita derecha.

\section{Discusión}

En nuestro estudio presentamos un método para estimar el volumen orbitario a partir de TC, utilizando el

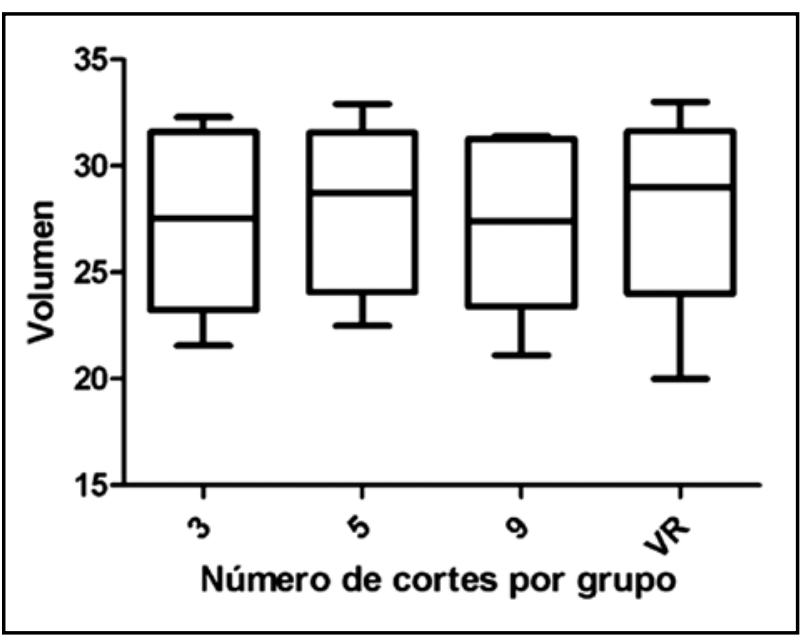

Figura 4. Diagrama de caja muestra el número de cortes $\mathrm{v} / \mathrm{s}$ volumen real de la órbita izquierda.

principio de Cavalieri, mediante la selección aleatoria de la primera imagen seguida de la observación de una secuencia predeterminada de imágenes. Este 
modelo, que combina un principio matemático (Cavalieri) con otro estadístico (aleatorización), representa una técnica simplificada de medición de volumen, de modo que no es necesario analizar la totalidad de las imágenes como lo realiza Acer et al ${ }^{(8)}$ y Bilgic et $\mathrm{al}^{(9)}$. El análisis se realiza sobre la pantalla del computador, lo que hace innecesaria la impresión de las imágenes, como hicieron Acer et $\mathrm{al}^{(8)}$, o la tenencia de las placas de TC, como en los estudios realizados por Charteris et $\mathrm{al}^{(3)}$ y Schuknecht et $\mathrm{al}^{(7)}$.

Nuestros resultados indican que es posible realizar una estimación válida del volumen orbitario a partir de un número pequeño de secciones observadas en la imagen de TC. Estos resultados no aumentan significativamente su exactitud al aumentar el número de imágenes observadas, no obstante el tiempo que un clínico deberá dedicar a la observación de las imágenes está en relación directa con el número de imágenes que va a analizar. Estos resultados concuerdan con lo indicado por Roberts et al ${ }^{(10)}$, quienes revisaron extensamente estos métodos y reportaron su validez para la evaluación del volumen de los tumores cerebrales, de la sustancia gris cerebral y el grosor medio del córtex.

Un aspecto crítico que afecta la validez de las observaciones es la configuración de la pantalla del computador donde se visualizan las imágenes. Las mediciones deben realizarse sobre la pantalla, manteniendo la relación vertical y horizontal de la imagen (resolución de pantalla 1280 x800) pues este aspecto técnico influye en la reproductibilidad y validez de las mediciones.

En TC convencional, el método propuesto de estimación del volumen orbitario puede ser realizado directamente sobre las placas de TC, pues se mantiene la relación entre los valores de LE y UE de la ecuación antes descrita. No se recomienda digitalizar las imágenes mediante fotografía, pues cualquier variación en la posición del foco se puede traducir en distorsiones que afectarían los resultados.

Para TC multicorte, se propone la elaboración de un software que en conjunto con la asociación de un visor de imágenes, permita la estimación del volumen de la órbita u otra región, delimitando y calculando el área de un número relativamente pequeño de imágenes de TC e incorporando las variables de la ecuación descrita en el método.

\section{Conclusión}

Hemos presentado un método confiable y sin sesgo matemático, para la determinación del volumen orbitario que puede ser utilizado con los medios disponibles, de manera rápida, a un bajo costo y de escasa dificultad técnica.

\section{Bibliografía}

1. Wang S, Xiao J, Liu L, Lin Y, Li X, Tang W et al. Orbital floor reconstruction: a retrospective study of 21 cases. Oral Surg Oral Med Oral Pathol Oral Radiol Endod 2008; 106: 324-330.

2. Metzger MC, Schön R, Schulze D, Carvalho C, Gutwald R, Schmelzeisen R. Individual preformed titanium meshes for orbital fractures. Oral Surg Oral Med Oral Pathol Oral Radiol Endod 2006; 102: 442-447.

3. Charteris DG, Chan $\mathrm{CH}$, Whitehouse RW, Noble JL. Orbital volume measurement in the management of pure blowout fractures of the orbital floor. $\mathrm{Br} \mathrm{J}$ Ophthalmol 1993; 77: 100-102.

4. Cooper WC. A method for volume determination of the orbit and its contents by high resolution axial tomography and quantitative digital image analysis. Trans Am Ophthalmol Soc 1985; 83: 546-609.

5. Ye J, Kook KH, Lee SY. Evaluation of computer-based volume measurement and porous polyethylene channel implants in reconstruction of large orbital wall fractures. Invest Ophthalmol Vis Sci 2006; 47:509-513.

6. Rose GE, Sigurdsson H, Collin R. The volume-deficient orbit: clinical characteristics, surgical management, and results after extraperiorbital implantation of Silastic block. Br J Ophthalmol 1990; 74: 545-550.

7. Schuknecht B, Carls F, Valavanis A, Sailer HF. CT assessment of orbital volume in late post-traumatic enophthalmos. Neuroradiology 1996; 38: 470-475.

8. Acer N, Bayar B, Basaloglu H, Oner E, Bayar K, Sankur $S$. Unbiased estimation of the calcaneus volume using the Cavalieri principle on computed tomography images. Ann Anat 2008; 190: 452-460.

9. Bilgic S, Sahin B, Sonmez OF, Odaci E, Colakoglu S, Kaplan $S$ et al. A new approach for the estimation of intervertebral disc volume using the Cavalieri principle and computed tomography images. Clin Neurol Neurosurg 2005; 107: 282-288.

10. Roberts N, Puddephat MJ, McNulty V. The benefit of stereology for quantitative radiology. $\mathrm{Br} \mathrm{J}$ Radiol 2000; 73: 679-697. 